\title{
Die Interstadiale der Weichselvereisung
}

Von Willi Selle, Braunschweig-Riddagshausen. Mit 2 Abb.

Im Laufe unserer Untersuchungen an Interglazialen wurden uns an drei Stellen des nordwestdeutschen Flachlandes Torfbänke bekannt, die, in Sanden eingebettet, sich im Hangenden von interglazialen Ablagerungen befinden. Auf einer Exkursion der Tagung der Arbeitsgemeinschaft Nordwestdeutscher Geologen im Jahre 1939 fanden wir über der Kieselgur von Schwindebeck zwei kleinere Torfbänke, von denen ich in demselben Jahre Proben entnahm und pollenanalytisch untersuchte. Während meiner Tätigkeit am Amt für Bodenforschung in Berlin erhielt ich Proben von einer Brunnenbohrung aus Örel bei Bremervörde. Die pollenanalytische Untersuchung zeigte, daß die aus dem Liegenden stammenden Torfe dem letzten Interglazial angehörten, während die Proben aus den beiden darüberliegenden Torfbänken, die je ca. $2 \mathrm{~m}$ mächtig waren, Pollendiagramme ergaben, die mit den Torfen von Schwindebeck und dem AllerödInterstadial gemeinsame Züge aufwiesen. Inzwischen hatten wir festgestellt, daß die Kieselgurlager des Luhetales zum letzten Interglazial gehören, so daß ich bereits damals vermutete, daß die Torfe von Schwindebeck und Örel Repräsentanten der Interstadiale des Brandenburger und Frankfurter und des Frankfurter und Pommerschen Stadiums der Weichselvereisung sein könnten. Bei der Abbohrung des Süßwassermergellagers von Nedden-Averbergen im Jahre 1942 stießen wir auf zwei Torfbänke von geringer Mächtigkeit, die sich im Hangenden der Ablagerungen des letzten Interglazials befanden. An dieser Stelle wurde eine Stoßkernbohrung niedergebracht, um einwandfreies Untersuchungsmaterial zu bekommen. Leider konnte seine Auswertung erst nach dem Kriege vorgenommen werden. Sie ergab eine gute Übereinstimmung mit den bisherigen Untersuchungen, so daß wir den Schluß ziehen können, daß die Torfbänke interstadiale Ablagerungen der Weichselvereisung sind.

Unsere Untersuchungen sind noch nicht abgeschlossen, da die Torfe von Schwindebeck mit den neueren Methoden der Pollenanalyse untersucht werden müssen. Aus der Brunnenbohrung von Örel erhielten wir Proben in größeren Abständen und ohne genauere Tiefenbezeichnung. Leider sind außerdem die Profilbeschreibung, die Proben und Präparate den Kriegswirren zum Opfer gefallen. Erhalten geblieben sind nur die Zählprotokolle und das Pollendiagramm. Wir planen daher, das wichtige Vorkommen von Örel durch eine erneute Bohrung zu erschließen.

Wenn wir bereits jetzt mit den vorläufigen Ergebnissen an die Öffentlichkeit treten, so geschieht es im Hinblick auf die lebhaften Erörterungen, die um die Gliederung der Würmeiszeit entstanden sind. BüDEL $(1949,1950,1951)$ teilt auf Grund seiner Untersuchungen die letzte Eiszeit in drei große Klimaphasen ein. Im Anfang herrschte ein kalt-ozeanisches Klima, das abgelöst wurde von einer kalt-kontinentalen Tönung, dem Hochstand der Gletscher in Mitteleuropa, dem die feucht-wärmere Schlußphase der Vereisung folgıe. Die Unterteilung in W I, W II und W III lehnt er ab, da nach seiner Ansicht die glazigenen und nichtglazigenen Ablagerungen der Würmeiszeit keinen Anhalt für Interstadiale, außer der Allerödzeit, ergeben haben. Sснӧnhals (1950, 1951a, 1951b) dagegen kommt durch seine Untersuchungen an Lößen zu dem Schluß, daß sich in den würmeiszeitlichen Lößen Verwitterungshorizonte befinden, die durch die Interstadiale W I/II und W II/III entstanden sind. Von pollenanalytischer Seite liegt ein neuerer Beitrag zu dieser Frage von Flonschürz aus den Niederlanden vor. 
Man nimmt ein Interstadial als gesichert an, über das zweite dagegen liegen keine eindeutigen Beweise vor (van der HAMmen 1951, van der VlekK \& FloRsCHÜтz 1950). Aus diesen wenigen Beispielen geht bereits hervor, wie widerspruchsvoll bis heute die Ansichten über die Gliederung der letzten Eiszeit sind. Wir nehmen an, daß durch die Untersuchung der Torfe über den Interglazialen von Schwindebeck, Örel und Nedden-Averbergen die Lösung der Unterteilung der Weichselvereisung wesentlich gefördert wird.

In dieser vorläufigen Mitteilung soll nur auf die Untersuchungsergebnisse von Örel und Nedden-Averbergen näher eingegangen werden. Diese beiden Vorkommen haben folgenden stratigraphischen Aufbau:

1. Örel bei Bremervörde

$0,00-6,00 \mathrm{~m}$ : Sand.

$6,00-8,00 \mathrm{~m}$ : Obere Torf.bank.

Probe 4-8: M $\quad$ u d d e: bräunlich-schwarz, stark zersetzt, sandig, vorwiegend organische Reste, Sphagnum- und Braunmoosblattfetzen, Seerosensternhaare, Pediastrum, Myriophyllum alterniflorum.

$8,00-10,00 \mathrm{~m}$ : Sand.

10,00 -12,00 m: Mittlere Torfbank.

Probe 12-17: Sphagnum-Carextorf: bräunlich-schwarz, stark zersetzt, sandig, Sphagnum- und Braunmoosreste, Carex-Wurzeln, Eriophorum, Pinus-Spaltöffnungen.

Probe 18-23: $\quad$ M u d d e: bräunlich-schwarz, stàrk zersetzt, sandig, vorwiegend organogen, Sphagnum- und Braunmoosreste, Carex-Wurzeln, Seerosensternhaare, Pediastrum, Myriophyllum alterniflorum.

$12,00-13,50 \mathrm{~m}:$ Sand.

$13,50-15,50 \mathrm{~m}$ : Untere Torfbank.

Probe 25: $\quad$ Carex-T orf: bräunlich-schwarz, stark zersetzt, sandig, Sphagnum-Blätter, Carex-Wurzeln.

Probe 26: $\quad$ Mudde: bräunlich-schwarz, stark zersetzt, Sphagnum-Reste, CarexWurzeln, Seerosensternhaare.

$15,50-16,50 \mathrm{~m}$ : Ton.

2. Nedden-Averbergen

$0,00-1,10 \mathrm{~m}$ : Torfe in Wechsellagerung mit Fein- bis Mittelsanden mit kleineren Geschieben; gestört durch Bewirtschaftung der Wiese. Holozän.

$1,10-1,50 \mathrm{~m}:$ Fein- bis Mittelsande mit kleineren Geschieben. Pleistozän.

1,50 - 1,60 m: M udde (Dy): braun-schwarz, H 10, sandig, Sphagnum-Reste, Carex-Wurzeln, Typha-Pollen.

$1,60-1,70 \mathrm{~m}$ : M udde (Dy): braun-schwarz, H 10, sandig, Sphagnum-Reste, Carex-Wurzeln, Seerosensternhaare. Typha-Pollen.

$1,70-2,105 \mathrm{~m}:$ Fein- bis Mittelsande mit vereinzelten kleineren Geschieben.

2,105- 2,15 m: M udd e (Dy): braun-schwarz, H 10, sandig, Sphagnum-Reste, Carex-Wurzeln, Gram.-Epidermis, Farnreste.

$2,15-2,225 \mathrm{~m}$ : M u d d e (Dy): braun-schwarz, H 10, sandig, wenig SphagnumReste, Gram.-Epidermis, Seerosensternhaare.

2,225-3,25 m: Fein- bis Mittelsande mit vereinzelten kleineren Geschieben.

$3,25-3,40 \mathrm{~m}$ : Sphagnum-Carex-Torf: braun-schwarz H 9/10, sandig, Sphagnum- und Braunmoosreste, Carex-Wurzeln, Eriophorum.

3,40-3,60 m: Car ex-T orf: H 7/8, bräunlich-schwarz, Carex-Wurzeln, Gram.Epidermis, Pinus-Spaltöffnungen, Birken- und Erlenholz und -rinde.

$3,60-3,65 \mathrm{~m}:$ Bruchwaldtorf: bräunlich-schwarz, H 7, Carex-Wurzeln, Gram.-Epidermis, Pinus-Spaltöffnungen, Birken- und Kiefernholz.

$\mathrm{Da}$ am Ende der Interglaziale mehrfach Umlagerungen und Verwehungen von Torfen festgestellt wurden, die durch das arktische Klima bedingt sind (Thомson 1951), mußte nachgeprüft werden, ob die Torfe über den Interglazialen von Örel und Nedden-Averbergen autochthon oder allochthon entstanden sind. Die Pollenkurven und der Aufbau der Torfe, die in beiden Interstadialen gleichsinnig verlaufen, lassen deutlich erkennen, daß die Mudden und Torfe sich in primärer Lagerung befinden. Die Mudden von Nedden-Averbergen haben einen dyartigen 


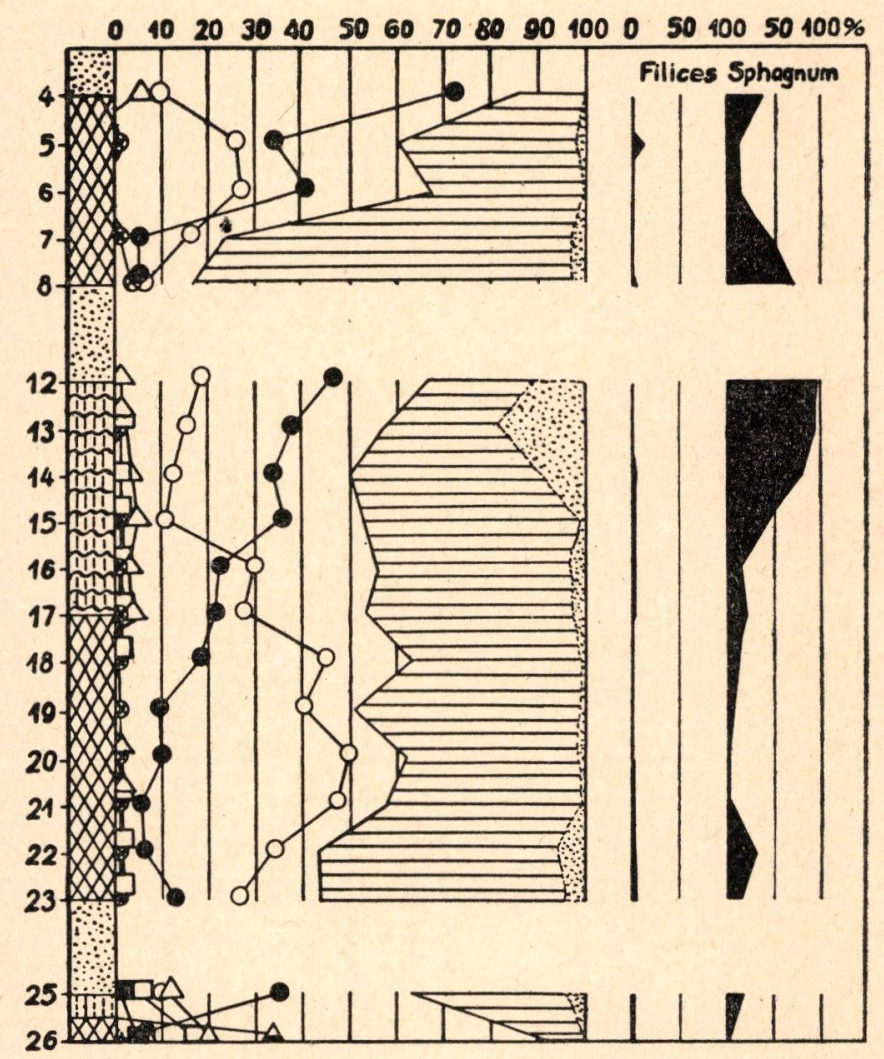

Abb. 1. Pollendiagramm von Örel.

\section{Legende}

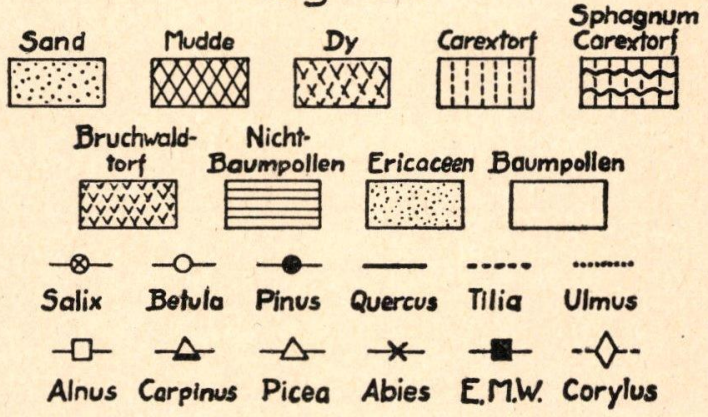

Charakter und sind nur gering mächtig, während sie in Örel einen größeren Umfang erreichen. Ob die Mudden von Örel aus Gyttjen oder Dy bestehen, ließ sich wegen der zerstörten Proben nicht mehr nachweisen. Das Fehlen von $\mathrm{Pe}$ diastrum und Myriophyllum in Nedden-Averbergen ist auf die örtlichen Verhältnisse zurückzuführen, die dem Tausendblatt und den Pediastren in dem flachen Sumpf keine günstigen Lebensbedingungen gaben. Die Ablagerungen be- 
stehen vorwiegend aus organischen Resten, sind aber durchgehend mit Fein- bis Mittelsanden durchsetzt. Der Verlauf der Verlandung ist in beiden Interstadialen derselbe. Anfangs sind Seggen und Farne stärker beteiligt, am Ende die oligotrophen Komponenten, wie z. B. Sphagnum und Eriophorum. In Örel schließt die Verlandung des ersten Interstadials mit einem Cariceto-Sphagnetum ab, das ebenfalls am Ende des Interglazials in Nedden-Averbergen bestand. Die Schluß-

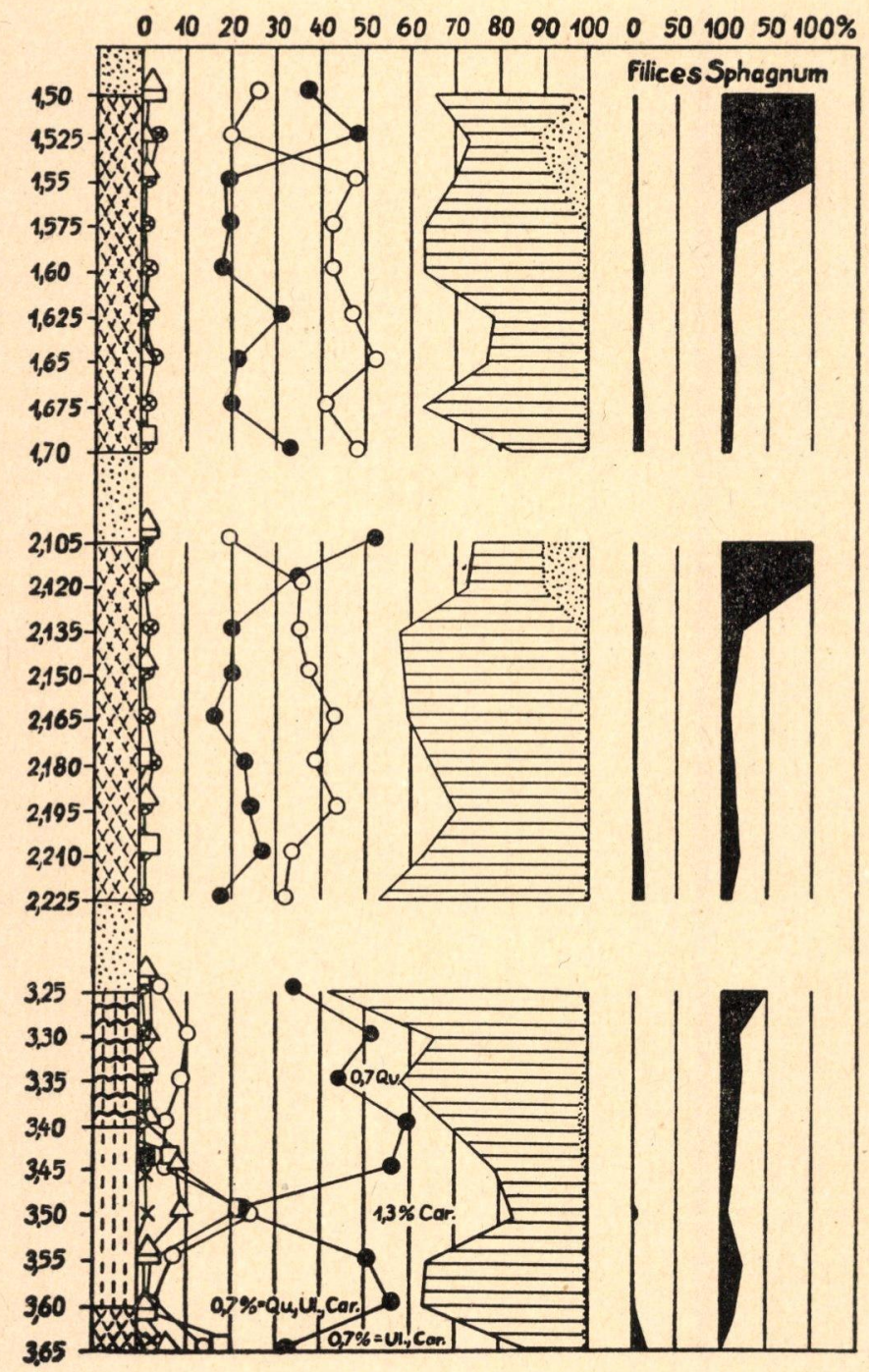

Ab. 2. Pollendiagramm von Nedden-Averbergen.

glieder der Verlandung in den beiden Interstadialen und dem letzten Interglazial weisen viele gemeinsame Züge auf, die sich nur aus ähnlichen klimatischen Verhältnissen erklären lassen. Die Rückläufigkeit der Verlandung im Interglazial von Nedden-Averbergen ist desgleichen klimatisch bedingt. Der Seggentorf, der dem Bruchwaldtorf folgt, ist zwar noch reichlich mit Bruchwaldelementen 
durchsetzt, zeigt aber die größere Vernässung an, die gegen das Ende des Interglazials eintrat (vgl. Firbas 1951, Weber 1896).

Die Zweiteilung der beiden Interstadiale, die in der Verlandung bereits hervortritt, findet ihre Bestätigung in den Pollendiagrammen, die mit einer Dominanz des Birkenpollens beginnen und mit einer hohen Kiefernpollenfrequenz enden (Abb. 1 und 2). Die Salixkurve hält sich mit wenigen Prozenten in beiden Interstadialen von Nedden-Averbergen, während sie in Örel zum Beginn einen höheren Gipfel aufweist, der mit einer größeren Beteiligung der NBP, vor allem der Gramineen, gekoppelt ist. Vermutlich sind in Örel durch die Bohrung die älteren Schichten erschlossen und in Nedden-Averbergen nicht, wo das Liegende aus Fein- bis Mittelsanden besteht. Diese hielten sich nicht im Stoßkernrohr und mußten mit der Schlammbüchse herausgeholt werden, wodurch eine mikroskopische Untersuchung unmöglich wurde. Außer Salix, Betula und Pinus erscheinen Alnus und Picea mehr oder weniger gleichmäßig im Pollenbilde. Über das Verhalten der Erle lassen sich keine Schlüsse ziehen; anzunehmen ist, daß sie in beiden Interstadialen an ihr zusagenden Orten in geringer Menge gestockt hat. Die Fichtenbeteiligung weist in beiden Interstadialen und an beiden Orten eine eindeutige Tendenz auf. Der Schwerpunkt ihrer Verbreitung lag am Ende der Interstadiale. Das Auftreten des Haselpollens bedarf einer Nachprüfung, da er außer von Corylus auch von Myrica gale stammen oder sekundären Ursprungs sein kann.

Die starke Ausbreitung der Birken und Kiefern, das regelmäßige Vorkommen der Weiden und das Fehlen der wärmeliebenden Bäume läßt auf ein subarktisches Klima schließen. Die NBP fügen sich gut in dieses Bild ein. Das Verhältnis der BP-Dichte und der der NBP pro Präparat $(18 / 18 \mathrm{~mm})$ beträgt im 1. Interstadial von Örel $108: 86$ und im 2. Interstadial $82: 124$, die entsprechenden Werte von Nedden-Averbergen sind 900:406 und 950:301 (Abb. 1 und 2). Die höheren NBP-Frequenzen in Örel sind auf die unteren Schichten zurückzuführen, in denen die kälteren Phasen der Interstadiale zum Ausdruck kommen, während die Ursache für die unterschiedliche Pollendichte in den Mudden und Torfen zu suchen ist. Die Werte der NBP sind nicht so hoch wie in Steppen, andererseits liegen sie höher als in Waldgebieten, so daß anzunehmen ist, daß in den Interstadialen fast geschlossene Wälder mit Birken und Kiefern herrschten. Das Vorkommen von Sanden in den Mudden und Torfen läßt ebenfalls erkennen, daß die Vegetationsdecke nicht geschlossen war. Das regelmäßige Auftreten von Artemisia in wenigen Prozenten weist in dieselbe Richtung und zeigt das Vorhandensein von steppenartigen Pflanzengesellschaften in geringer Ausdehnung an. In den Proben von Örel sind die Pollen von Artemisia nicht ausgeschieden. Die anfangs grasreichen Birken-Kiefernwälder wurden später von zwergstrauchreichen Kiefern-Birkenwäldern abgelöst. Unter den Ericaceen-Pollen nehmen Calluna und Empetrum den größten Raum ein, der Vaccinium-Typ dagegen kommt nur spärlich vor. Die Farnsporen stammen zum größten Teil aus den Mooren, da in der Regel mit ihnen in den Ablagerungen Reste von Farnen auftreten. Ihr häufiges Erscheinen in den flachen Sümpfen von Nedden-Averbergen und ihr geringes Vorkommen in dem tieferen Becken von Örel zeigen ebenfalls ihre Bindung an die Verlandungsgesellschaften an. Ähnlich liegen die Verhältnisse im letzten Drittel der Interglaziale. Die Farnsporen gehören vorwiegend dem Dryopteris-Typ an, und nur in wenigen Fällen konnte der Polypodium-Typ festgestellt werden. 
Das subarktische Klima wird weiter durch die geringen Werte von Hippophaë rhamnoides bestätigt. Seine größte Ausbreitung erreicht der Sanddorn in der älteren Dryaszeit kurz vor dem Beginn der Bewaldung, so daß sich sein geringes Vorkommen gut in das Bild einfügt, das wir durch die anderen Pflanzen erhalten haben (Frrbas 1949). Helianthemum-Pollen fehlen in den Interstadialen von Nedden-Averbergen, nur einmal fand er sich in der letzten Probe des darunterliegenden Interglazials. Die Nachprüfung für Örel mußte wegen der Vernichtung des Materials durch den Krieg unterbleiben. Aus dem Verhalten von Myriophyllum alterniflorum und Empetrum nigrum geht hervor, daß das subarktische Klima eine ozeanische Tönung besessen haben muß. Das Schwergewicht der Ausbreitung von Empetrum scheint am Ende der Interstadiale gelegen zu haben. Es wird damit die Ansicht bestätigt, daß die Vereisungen durch ein niederschlagsreiches (vermutlich schneereiches), feucht-kühles Klima eingeleitet wurden (vgl. Büdel 1949, Firbas 1951, Poser 1948a und 1948b). An den Profilen von Örel und Nedden-Averbergen kann dieser Vorgang dreimal verfolgt werden, der in den Pollendiagrammen durch den Anstieg der Kiefern-, Fichten-, Empetrum-, Calluna- und Sphagnum-Kurven zum Ausdruck kommt. Diese Klimaänderung spiegelt sich ebenfalls in den Mudden und Torfen wider, die zunehmend oligotroph werden.

In mancher Hinsicht kehren diese Verhältnisse in der Alleröd-Oszillation wieder, wo sie allerdings wegen des schwächeren Klimarückschlages der jüngeren Dryaszeit nicht so klar in Erscheinung treten. Das Alleröd-Interstadial weist viele gemeinsame Züge mit den beiden Interstadialen auf, wie z. B. die Unterteilung in eine Birken- und Kiefern-Phase und das Verhältnis der BP zu den NBP (Tabelle 1). Sie unterscheiden sich aber auch, so daß sie gegeneinander abgegrenzt werden können. Die Interstadiale hatten eine größere Ozeanität, wodurch Empetrum und Myriophyllum alterniflorum hier häufiger vorkommen als in der Allerödzeit. Ein weiteres Unterscheidungsmerkmal ist das Auftreten von Picea in den Interstadialen, die gewöhnlich dem Spätglazial fehlt. In den meisten Alleröd-Ablagerungen sind Farnsporen selten, während sie in den interstadialen Diagrammen regelmäßig erscheinen.

Unter Varia befinden sich $u$. a. einige Pollen von Umbelliferen und Kompositen. Es muß damit gerechnet werden, daß das geringe Vorkommen von Pollen einer Art, wie z. B. Hippophaë und Helianthemum, auf sekundäre Einlagerung zurückzuführen ist. Ein eindeutiges Bild wird sich erst aus weiteren Untersuchungen ergeben.

Ebenso regelmäßig wie das Ende der Interglaziale und Interstadiale durch den Anstieg der Pinuskurve gekennzeichnet ist, tritt mit der beginnenden Klimaverbesserung in der gleichen Gesetzmäßigkeit eine starke Zunahme der Birke auf. An sämtlichen Pollendiagrammen der Interglaziale, der beiden würmeiszeitlichen Interstadiale, der Alleröd-Oszillation und der Nacheiszeit können diese Vorgänge beobachtet werden. Es müssen demnach grundsätzliche Unterschiede zwischen den Übergängen von einer Warmzeit zu einer Eiszeit und von einer Vereisung zu einer Wiedererwärmung bestanden haben. Die beginnende Eiszeit war vermutlich durch ein feucht-kühles, schneereiches Klima gekennzeichnet, in der die Niederschläge in Gletschern festgelegt wurden, während am Ende der Eiszeit die Gletscher abschmolzen, wodurch viel Wärme verbraucht wurde. Es entstanden Vegetationsperioden, die sich in Länge und Wärme unterschieden. In den Birkenphasen war das Klima wahrscheinlich kühler und die Vegetationszeit länger als in den Kiefernphasen. 


\begin{tabular}{|c|c|c|c|c|c|c|c|c|c|c|c|c|c|c|c|c|c|c|c|}
\hline & & 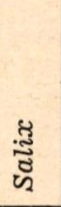 & 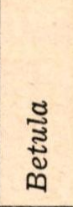 & 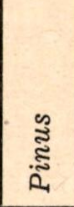 & స్ & $\begin{array}{l}\tilde{\Xi} \\
\tilde{\pi} \\
\tilde{z}\end{array}$ & 胥 & $\begin{array}{l}\text { Pi } \\
\text { mi } \\
\text { zi }\end{array}$ & 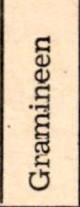 & 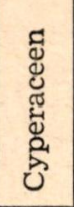 & 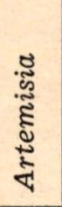 & 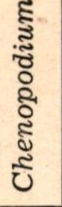 & 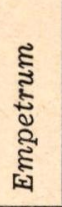 & 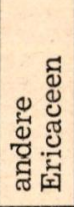 & 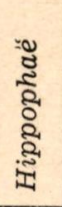 & 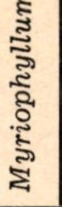 & 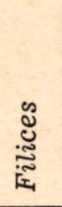 & 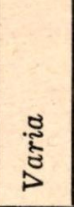 & 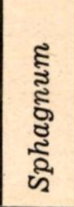 \\
\hline \multirow{6}{*}{$\begin{array}{c}\text { Birken- } \\
\text { phase }\end{array}$} & 1. Interstadial von Orel & 3,3 & $72,-$ & 22,3 & 1,3 & 1,1 & - & 90,4 & 58,2 & 7,1 & $?$ & ? & 1,7 & 3,7 & - & 0,1 & 2,5 & 17,1 & 13,4 \\
\hline & $\begin{array}{l}\text { 1. Interstadial } \\
\text { von Nedden-Averbergen }\end{array}$ & 2,6 & $62,-$ & 34,9 & 0,9 & 0,2 & 0,4 & 66,2 & 28,6 & 19,2 & 2,7 & 0,7 & 0,4 & 0,4 & - & - & 7,8 & 6,8 & 14,1 \\
\hline & 2. Interstadial von Orel & 8,8 & 69,4 & 21,8 & - & - & - & $250,-$ & $196,-$ & $7,-$ & $?$ & $?$ & - & 7,5 & 0,3 & 6,5 & $2,-$ & 29,5 & 56,5 \\
\hline & $\begin{array}{l}\text { 2. Interstadial } \\
\text { von Nedden-Averbergen }\end{array}$ & 1,9 & 65,2 & 32,6 & - & 0,3 & 0,2 & 41,8 & 16,1 & 9,5 & $2,-$ & - & 0,4 & 1,7 & 0,2 & - & 4,9 & 6,9 & 32,3 \\
\hline & $\begin{array}{l}\text { Alleröd-Interstadial } \\
\text { von Stellmoor }\end{array}$ & 2,0 & 56,0 & 41,7 & - & - & - & 41,7 & $20,-$ & $15,-$ & $?$ & $?$ & - & $1,-$ & - & - & - & 5,7 & $?$ \\
\hline & $\begin{array}{l}\text { Alleröd-Interstadial } \\
\text { von Dannenberg }\end{array}$ & 3,8 & 89,1 & 7,0 & - & + & - & 62,6 & 12,5 & 34,3 & ? & ? & 1,5 & - & - & + & - & 14,3 & $?$ \\
\hline \multirow{6}{*}{$\begin{array}{c}\text { Kiefern- } \\
\text { phase }\end{array}$} & 1. Interstadial von Orel & $1,-$ & 30,6 & $63,-$ & $1,-$ & 4,4 & - & 80,6 & 51,6 & 8,4 & $?$ & $?$ & 2,2 & 12,6 & - & - & 0,8 & $5,-$ & 65,2 \\
\hline & $\begin{array}{l}\text { 1. Interstadial } \\
\text { von Nedden-Averbergen }\end{array}$ & $1,-$ & 37,2 & 58,8 & 0,5 & 2,5 & - & $35,-$ & 6,5 & 5,5 & 0,5 & 1,0 & 2,5 & 10,5 & - & - & 1,5 & $7,-$ & 131,5 \\
\hline & 2. Interstadial von Orel & 0,4 & 31,3 & 66,3 & - & $2,-$ & - & 44,7 & $27,-$ & 3,7 & $?$ & $?$ & 0,7 & 0,3 & - & 0,3 & 4,7 & $8,-$ & 21,6 \\
\hline & $\begin{array}{l}\text { 2. Inferstadial } \\
\text { von Nedden-Averbergen }\end{array}$ & 2,8 & 33,3 & 61,9 & 1,0 & 1,0 & - & 46,7 & $26,-$ & 3,4 & 0,5 & - & $3,-$ & 6,9 & - & - & 0,9 & $6,-$ & 144 \\
\hline & $\begin{array}{l}\text { Alleröd-Interstadial } \\
\text { von Stellmoor }\end{array}$ & 3,3 & 32,4 & 64,7 & - & - & - & 53,6 & 21,8 & 22,2 & $?$ & $?$ & - & 0,2 & - & - & - & 9,4 & $?$ \\
\hline & $\begin{array}{l}\text { Alleröd-Interstadial } \\
\text { von Dannenberg }\end{array}$ & 1,7 & 55,5 & 42,8 & - & - & - & 40,6 & 9,8 & 22,2 & $?$ & $?$ & 1,2 & 2,4 & - & - & - & $5,-$ & $?$ \\
\hline
\end{tabular}




\section{$\mathrm{Zusammenfassung}$}

An drei Orten im nordwestdeutschen Flachlande konnten zwei Interstadiale der Weichseleiszeit festgestellt werden. Vermutlich gehören die Torfe oberhalb des Interglazials von Eversen bei Rotenburg/Hann. ebenfalls einem dieser Interstadiale an (WolfF \& SchröDER 1940).

Die stratigraphischen Verhältnisse lassen eine eindeutige Eingliederung in die Weichselvereisung $\mathrm{zu}$. In allen Fällen liegen die beiden Torfbänke oberhalb des letzten Interglazials und sind in Sanden der letzten Eiszeit eingeschlossen. Von einer Parallelisierung mit den Stadien der Weichselvereisung soll vorläufig Abstand genommen werden.

Durch die Pollenanalyse ergab sich eine Unterteilung in eine Birken- und in eine Kiefernphase. Das Klima war subarktisch-ozeanisch. Die Wiedervereisung nach dem letzten Interglazial und den beiden Interstadialen wurde durch ein feucht-kühles, niederschlagsreiches (vermutlich schneereiches) Klima eingeleitet.

Beide Interstadiale haben mit der Alleröd-Oszillation gemeinsame Züge, lassen sich jedoch klar von ihr trennen.

$\mathrm{Ob}$ eine Unterscheidung der beiden Interstadiale mit Hilfe der Pollenanalyse vorgenommen werden kann, ließen die bisherigen Untersuchungen nicht erkennen und muß daher den weiteren Arbeiten vorbehalten bleiben.

\section{Schriftum}

BüDEL, J.: Die räumliche und zeitliche Gliederung des Eiszeitklimas. - Die Naturwissenschaften 36, S. 105-112, 133-139. 1949. - Die Klimaphasen der Würmeiszeit. Ebenda 37, S. 438-449, 1950. - Die Klimazonen des Eiszeitalters. - Eiszeitalter und Gegenwart 1, S. 16-26. 1951.

Firbas, F.: Waldgeschichte Mitteleuropas, I. Bd. 1949. - Die quartäre Vegetationsentwicklung zwischen den Alpen und der Nord- und Ostsee. - Erdkunde 5, S. 6-15. 1951.

Hammen, van DeR, Th.: Late-glazial flora and periglazial phenomena in the Nederlands. Leidse Geol. Mededel. 17, S. 71-183. 1951.

Overbeck, F.: Ein spätglaziales Profil von Huxfeld bei Bremen. - Planta 37, S. 376-389. 1949.

Poser, H.: Aeolische Ablagerungen und Klima des Spätglazials in Mittel- und Westeuropa. - Die Naturwissenschaften 35, S. 269-276, 307-312. 1948a. - Bodenund Klimaverhältnisse in Mittel- und Westeuropa während der Würmeiszeit. - Erdkunde 2, S. 53-68. 1948b.

Schönhals, E.: Über einige wichtige Lößprofile und begrabene Böden im Rheingau. Notizblatt des Hessischen Landesamtes für Bodenforschung 6, H. 1, S. 244259. 1950. - Fossile gleiartige Böden des Pleistozäns im Usinger Becken und am Rande des Vogelsbergs. - Ebenda 6, H. 2, S. 160-183. 1951 a. - Über fossile Böden im nichtvereisten Gebiet. - Eiszeitalter und Gegenwart 1, S. $109-130.1951 \mathrm{~b}$.

Thomson, P. W.: Das Interglazial von Wallensen im Hils. - Eiszeitalter und Gegenwart 1, S. 96-102. 1951.

VAN DER Vlerk, J. M. \& Florschütz, F.: Nederland in het ijstijdvak. - Utrecht 1950.

WEBER, C. A.: Über die fossile Flora von Honerdingen und das nordwestdeutsche Diluvium. - Abh. nat. Ver. Bremen 13, S. 413-468. 1896.

WolfF, W. \& SCHRöDER, D.: Interglazialbildungen von Eversen bei Rotenburg i. Hann. Jb. d. Moorkunde 27, S. 7-19. 1940. 